\title{
Conservation of Flux in Superradiance Phenomenon
}

\author{
Petarpa Boonserm, Tritos Ngampitipan, and Matt Visser
}

\begin{abstract}
For a usual occurrence of wave scattering, the amplitude of the reflected wave is less than that of the incident wave because the incident wave loses energy to the reflective obstacle. However, for the so-called superradiance phenomenon, the amplitude of the reflected wave is more than that of the incident wave since the incident wave extracts energy from the reflective obstacle. In this paper, a simple toy model of superradiance is presented. The results show that for the case of superradiance, we derive a conservation of flux instead of the conservation of probability.
\end{abstract}

Index Terms - Conservation, flux, probability, superradiance.

\section{INTRODUCTION}

The phenomena of scattering can be described by the interaction of wave with a reflective physical obstacle. In a general situation, the incident wave loses some of its energy to the obstacle, resulting in the amplitude of the reflected wave being less than that of the incident wave. However, in some systems, the incident wave gains energy from the obstacle instead of losing energy. Therefore, the amplitude of the reflected wave becomes greater than that of the incident wave. This unusual phenomenon is called superradiance. Matters of superradiance in literature can be found in [1]-[20].

Despite a long scientific history, superradiance still generates some degree of confusion. Part of the confusion comes from a lack of understanding of the differences between fluxes and probabilities. In this paper, a simple toy model of superradiance is presented to clarify the concept.

\section{SUPERRADIANCE}

In non-relativistic quantum mechanics, superradiance does not take place [21]. To see this, consider the Schrödinger equation

$$
i \hbar \partial_{t} \psi(x, t)=-\frac{\hbar^{2}}{2 m} \partial_{x}^{2} \psi(x, t)+V(x) \psi(x, t) .
$$

Assuming the solution

Manuscript received October 3, 2014; revised January 13, 2015.

P. Boonserm is with the Department of Mathematics and Computer Science, Faculty of Science, Chulalongkorn University, Bangkok 10330, Thailand (e-mail: petarpa.boonserm@gmail.com).

T. Ngampitipan is with the Particle Physics Research Laboratory, Department of Physics, Faculty of Science, Chulalongkorn University, Bangkok 10330, Thailand (e-mail: tritos.ngampitipan@gmail.com).

M. Visser is with the School of Mathematics, Statistics, and Operations Research, Victoria University of Wellington, PO Box 600, Wellington, New Zealand (e-mail: matt.visser@msor.vuw.ac.nz).

$$
\psi(x, t)=e^{-i \omega t / \hbar} \psi(x)
$$

The Schrödinger equation becomes

$$
\frac{\hbar^{2}}{2 m} \partial_{x}^{2} \psi(x)=[V(x)-\omega] \psi(x) .
$$

On the other hand, in the relativistic regime we have the Klein-Gordon equation

$$
\left\lfloor-\left(\partial_{t}-i \varpi(x)\right)^{2}+\partial_{x}^{2}-V(x)\right\rfloor \psi(t, x)=0 .
$$

For a neutral scalar field, we assume the solution

$$
\psi(x, t)=e^{-i \omega t} \psi(x)
$$

The Klein-Gordon equation becomes

$$
\partial_{x}^{2} \psi(x)=\left[V(x)-\{\omega-\varpi(x)\}^{2}\right] \psi(x)
$$

In this case, superradiance can occur. We see that the term $[\omega-\varpi(x)]^{2}$ is responsible for superradiance. For a charged scalar field, we obtain

$$
\partial_{x}^{2} \psi(x)=\left[V(x)-\{\omega-\varpi(x)-q \Phi(x)\}^{2}\right] \psi(x),
$$

where $q$ is the charge of the scalar field. The term $[\omega-\varpi(x)-q \Phi(x)]^{2}$ is also responsible for superradiance.

\section{FluXES IN SUPERRADIANCE PHENOMENON}

In ordinary phenomena of wave scattering, we are familiar with the term 'probability' through both 'reflection probability' and 'transmission probability'. For a more general situation, including the case of superradiance, it is preferable to calculate the quantities in terms of fluxes rather than probabilities. The general conservation law can be described by

$$
F_{\text {reflected }}+F_{\text {transmitted }}=1-F_{\text {dissipated }} \text {. }
$$

In this paper, we are interested in cases of non-dissipation, where $F_{\text {dissipated }}=0$. The general cases, including dissipation, can be found in [21]. In ordinary cases, if the transmitted flux is non-negative $F_{\text {transmitted }} \geq 0$, it can be reduced to transmission probability $F_{\text {transmitted }}=T$. Moreover, the reflected flux also reduces to reflection probability $F_{\text {reflected }}=R$. Therefore (8) becomes 


$$
R+T=1
$$

This is the familiar conservation law of probabilities. On the other hand, in the case of superradiance, we have $F_{\text {transmitted }}<0$. It cannot be interpreted as the transmission probability. Thus, in any situation, we should work with quantities in terms of fluxes rather than probabilities.

\section{TOY MODEL FOR SUPERRADIANCE}

Consider the Klein-Gordon equation in 1+1 dimensions

$$
\left\lfloor-\left(\partial_{t}-i \varpi(x)\right)^{2}+c^{2} \partial_{x}^{2}-V(x)\right\rfloor \psi(t, x)=0 .
$$

Assuming the solution $\psi(t, x)=e^{-i \omega t} \psi(x)$, we obtain

$$
c^{2} \partial_{x}^{2} \psi(x)=\left[V(x)-\{\omega-\varpi(x)\}^{2}\right] \psi(x)
$$

Now, we simplify the problem by letting $V(x) \rightarrow 0$ and taking

$$
\varpi(x)=\Omega \operatorname{sign}(x),
$$

where $\Omega$ is a constant. Moreover, we set $c=1$. Therefore, (11) becomes

$$
\partial_{x}^{2} \psi(x)=-[\omega-\Omega \operatorname{sign}(x)]^{2} \psi(x)
$$

The solutions to (13) are given by

$$
\psi(x)=\left\{\begin{array}{lll}
e^{i k_{-} x}+r e^{-i k_{-} x} & \text { for } & x<0 \\
t e^{i k_{+} x} & \text { for } & x>0
\end{array},\right.
$$

where $r$ is the reflection amplitude, $t$ is the transmission amplitude, and

$$
k_{ \pm}^{2}=(\omega \mp \Omega)^{2} \text {. }
$$

Note that

$$
k_{+} k_{-}=\omega^{2}-\Omega^{2}
$$

Thus, we obtain

$$
\operatorname{sign}\left(k_{+} k_{-}\right)=\operatorname{sign}\left(\omega^{2}-\Omega^{2}\right)
$$

Assuming that wave moves from left to right and crosses the border at the origin, we have

$$
e^{i k_{-} x}+r e^{-i k_{-} x \breve{\mathrm{S}}} \quad t e^{i k_{+} x} .
$$

The continuity of the wave function leads to

$$
1+r=t
$$

The continuity of the derivative of the wave function leads to

$$
k_{-}(1-r)=k_{+} t
$$

Solving the equations, we obtain

$$
1+r=\frac{k_{-}}{k_{+}}(1-r)
$$

Rearranging it gives

$$
r=-\frac{k_{+}-k_{-}}{k_{+}+k_{-}}=-\frac{(\omega-\Omega)-(\omega+\Omega)}{(\omega-\Omega)+(\omega+\Omega)}=+\frac{\Omega}{\omega} .
$$

Since the reflection amplitude is normalization independent, the result is valid. The reflected flux is given by

$$
F_{\text {reflected }}=|r|^{2}=\frac{\Omega^{2}}{\omega^{2}} .
$$

However, the transmission amplitude depends on the normalization. For the relativistic Klein-Gordon equation, the normalization factor is

$$
\frac{e^{i k_{-} x}}{\sqrt{2\left|k_{-}\right|}}
$$

Therefore, the normalized solutions to (13) are given by

$$
\psi(x)=\left\{\begin{array}{ll}
\frac{e^{i k_{-} x}}{\sqrt{2\left|k_{-}\right|}}+\frac{r e^{-i k_{-} x}}{\sqrt{2\left|k_{-}\right|}} & \text {for } \quad x<0 \\
\frac{t e^{i k_{+} x}}{\sqrt{2\left|k_{+}\right|}} & \text {for } x>0
\end{array} .\right.
$$

The continuity of the wave function leads to

$$
\frac{1+r}{\sqrt{2\left|k_{-}\right|}}=\frac{t}{\sqrt{2\left|k_{+}\right|}}
$$

The continuity of the derivative of the wave function leads to

$$
\frac{k_{-}}{\sqrt{2\left|k_{-}\right|}}(1-r)=\frac{k_{+}}{\sqrt{2\left|k_{+}\right|}} t
$$

Solving the equations, we obtain

$$
\frac{1+r}{\sqrt{2\left|k_{-}\right|}}=\frac{k_{-}}{k_{+}} \frac{1-r}{\sqrt{2\left|k_{-}\right|}}
$$

Rearranging it gives

$$
r=-\frac{k_{+}-k_{-}}{k_{+}+k_{-}}=-\frac{(\omega-\Omega)-(\omega+\Omega)}{(\omega-\Omega)+(\omega+\Omega)}=+\frac{\Omega}{\omega} .
$$

Substituting in (26), we obtain 


$$
t=\sqrt{\frac{\left|k_{+}\right|}{\left|k_{-}\right|}}\left(1+\frac{\Omega}{\omega}\right)=\sqrt{\frac{|\omega-\Omega|}{|\omega+\Omega|}}\left(\frac{\omega+\Omega}{\omega}\right) .
$$

The reflected flux is given by

$$
F_{\text {reflected }}=|r|^{2}=\frac{\Omega^{2}}{\omega^{2}} .
$$

If $|\omega|>|\Omega|$, we have

$$
\begin{aligned}
& t=\sqrt{\frac{\omega-\Omega}{\omega+\Omega}}\left[\frac{\omega+\Omega}{\omega}\right]=\frac{\sqrt{\omega^{2}-\Omega^{2}}}{\omega} \\
& =\operatorname{sign}(\omega) \sqrt{1-\frac{\Omega^{2}}{\omega^{2}}} .
\end{aligned}
$$

Therefore, the transmitted flux is given by

$$
|t|^{2}=1-\frac{\Omega^{2}}{\omega^{2}} \geq 0
$$

We see that

$$
F_{\text {reflected }}+|t|^{2}=1
$$

In this case, we can write

$$
F_{\text {transmitted }}=|t|^{2} \geq 0 \text {. }
$$

On the other hand, if $|\omega|<|\Omega|$, we have

$$
\begin{aligned}
t & =\sqrt{-\frac{(\omega-\Omega)}{(\omega+\Omega)}}\left(\frac{\omega+\Omega}{\omega}\right)=\frac{\sqrt{\Omega^{2}-\omega^{2}}}{\omega} \\
& =\operatorname{sign}(\omega) \sqrt{\frac{\Omega^{2}}{\omega^{2}}-1}
\end{aligned}
$$

The transmitted flux is given by

$$
|t|^{2}=\frac{\Omega^{2}}{\omega^{2}}-1
$$

We see that

$$
F_{\text {reflected }}-|t|^{2}=1
$$

In this case, we can write

$$
F_{\text {transmitted }}=-|t|^{2} \leq 0 .
$$

We summarize both the cases by

$$
F_{\text {transmitted }}=\operatorname{sign}\left(k_{+} k_{-}\right)|t|^{2}=1-\frac{\Omega^{2}}{\omega^{2}} .
$$

Thus, we can write

$$
F_{\text {reflected }}+F_{\text {transmitted }}=1 \text {. }
$$

Using (17), this can be rewritten as

$$
|r|^{2}+\operatorname{sign}\left(k_{+} k_{-}\right)|t|^{2}=1 \text {. }
$$

Explicitly, this is not a conservation of probability, but rather, a conservation of flux.

\section{CONCLUSION}

Superradiance is a phenomenon of scattering in which the amplitude of the reflected wave is more than that of the incident wave because the incident wave extracts energy from the reflective obstacle. In this paper, a simple toy model of superradiance has been presented. In the case of superradiance, we have achieved the conservation of flux instead of the conservation of probability. The concept of conservation of probability is only valid in the absence of superradiance. So, in any situation (both with and without superradiance) we can write the conservation of flux

$$
F_{\text {reflected }}+F_{\text {transmitted }}=1
$$

if there is no dissipation. This can be rewritten as

$$
|r|^{2}+\operatorname{sign}\left(k_{+} k_{-}\right)|t|^{2}=1 \text {. }
$$

\section{ACKNOWLEDGMENT}

This project was funded by Ratchadapisek Sompoch Endowment Fund, Chulalongkorn University (Sci-Super 2014-032), by a grant for the professional development of new academic staff from the Ratchadapisek Somphot Fund at Chulalongkorn University, by the Thailand Toray Science Foundation (TTSF), by the Thailand Research Fund (TRF), by the Office of the Higher Education Commission (OHEC), Faculty of Science, Chulalongkorn University (MRG5680171), and by the Research Strategic plan program (A1B1). PB was additionally supported by a scholarship from the Royal Government of Thailand. TN was also supported by a scholarship from the Development and Promotion of Science and Technology talent project (DPST). MV was supported by the Marsden Fund, and by a James Cook fellowship, both administered by the Royal Society of New Zealand.

\section{REFERENCES}

[1] Y. B. Zel'dovich, "Amplification of cylindrical electromagnetic waves reflected from a rotating body," Journal of Experimental and Theoretical Physics, vol. 35, pp. 1085-1087, December 1972.

[2] C. A. Manoge, "The Klein paradox and super-radiance," Annals of Physics, vol. 181, pp. 261-283, February 1988.

[3] M. Richartz, S. Weinfurtner, A. J. Penner, and W. G. Unruh, "General universal super-radiant scattering," Physical Review D, vol. 80, December 2009.

[4] M. Richartz, A. Prain, S. Weinfurtner, and S. Liberati, "Super-radiant scattering of dispersive fields," Classical and Quantum Gravity, vol. 30, March 2013.

[5] A. Hansen and F. Ravndal, "Klein's paradox and its resolution," Physica Scripta, vol. 23, January 1981.

[6] N. Dombey and A. Calogeracos, "Seventy years of the Klein paradox," Physics Reports, vol. 315, pp. 41-58, July 1999.

[7] A. Calogeracos and N. Dombey, "History and physics of the Klein paradox," Contemporary Physics, vol. 40, pp. 313-321, 1999.

[8] W. H. Press and S. A. Teokolsky, "Floating orbits, super-radiant scattering and the black-hole bomb," Nature, vol. 238, pp. 211-212, July 1972 .

[9] E. Winstanley, "On classical super-radiance in Kerr-Newman - anti-de Sitter black holes," Physical Review D, vol. 64, October 2001.

[10] V. Cardoso, O. J. C. Dias, J. P. S. Lemos, and S. Yoshida, "The black hole bomb and super-radiant instabilities," Physical Review D, vol. 70, August 2004. 
[11] S. R. Dolan, "Instability of the massive Klein-Gordon-field on the Kerr spacetime," Physical Review D, vol. 76, October 2007.

[12] S. Chen and J. Jing, "Phantom scalar emission in the Kerr black hole spacetime," Classical and Quantum Gravity, vol. 27, January 2010.

[13] M. Kenmoku. (May 2009). Normal modes, quasi-normal modes and super-radiant modes for scalar fields in Kerr anti-de Sitter spacetime. Available: [Online]. http://www. arxiv.org/pdf/0809.2634v3.pdf

[14] S. R. Dolan, "Super-radiant instabilities of rotating black holes in the time domain," Physical Review D, vol. 87, June 2013.

[15] C. A. R. Herdeiro, J. C. Degollado, and H. F. Runarsson, "Rapid growth of super-radiant instabilities for charged black holes in a cavity," Physical Review D, vol. 88, September 2013.

[16] S. Hod, "Analytic treatment of the charged black-hole-mirror bomb in the highly explosive regime," Physical Review $D$, vol. 88, September 2013.

[17] J. C. Degollado and C. A. R. Herdeiro, "Time evolution of superradiant instabilities for charged black holes in a cavity," Physical Review D, vol. 89, March 2014.

[18] P. Boonserm, T. Ngampitipan, and M. Visser, "Bounding the greybody factors for scalar field excitations of the Kerr-Newman spacetime," Journal of High Energy Physics, pp. 113, March 2014.

[19] P. Boonserm, A. Chatrabhuti, T. Ngampitipan, and M. Visser "Greybody factors for Myers-Perry black holes," Journal of Mathematical Physics, vol. 55, November 2014.

[20] T. Ngampitipan, P. Boonserm, A. Chatrabhuti, and M. Visser, "Spin-zero Hawking radiation: Bounds on the zero-angular-momentum mode emission from Myers-Perry black holes," in Proc. the International Conference on Mechanical and Aerospace Engineering, 2014.

[21] P. Boonserm, T. Ngampitipan, and M. Visser, "Superradiance and flux conservation," Physical Review D, vol. 90, September 2014.

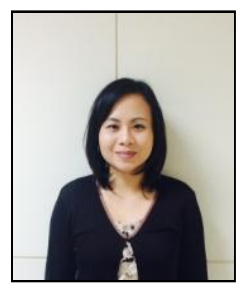

Petarpa Boonserm received the BSc., MSc., and Ph.D. degrees in mathematics from Victoria University of Wellington, Wellington, New Zealand, in 2004, 2006, and 2009, respectively.

She is an assistant professor at the Department of Mathematics and Computer Science, Faculty of Science, Chulalongkorn University, Bangkok, Thailand. Her current research interests include theory, and general relativity.

differential equations, applied mathematics, quantum

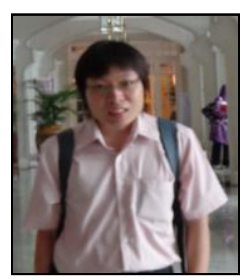

Tritos Ngampitipan received the B.Sc., M.Sc., and $\mathrm{Ph} . \mathrm{D}$. degrees in physics from Chulalongkorn University, Bangkok, Thailand, in 2008, 2011, and 2015 , respectively.

$\mathrm{He}$ is a researcher at the Department of Physics, Faculty of Science, Chulalongkorn University, Bangkok, Thailand. His current research interests include quantum theory and general relativity.

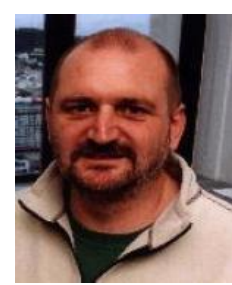

Matt Visser received the Ph.D. degree from University of California at Berkeley, California, USA.

$\mathrm{He}$ is a professor at the School of Mathematics, Statistics, and Operations Research, Victoria University of Wellington, Wellington, New Zealand. $\mathrm{He}$ is best known for his contributions to the theory of traversable wormholes, chronology protection, and analogue spacetimes. His research interests include general relativity, quantum field theory, and cosmology. 